\title{
The myth of the normal eye: A methodological note
}

\author{
STANLEY COREN \\ University of British Columbia, Vancouver, Canada V6T 1 W5 \\ and \\ CLARE PORAC \\ University of Victoria, Victoria, Canada
}

\begin{abstract}
A survey of 264 articles, investigating basic visual functioning indicates that nearly $72 \%$ do not report any visual screening of subjects. This data is compared with actual acuity testing of 111 avowedly visually "normal" observers, who did not wear corrective lenses, to point out the necessity of prescreening subjects in any paradigm where visual stimuli are to be used.
\end{abstract}

We recently conducted a series of experiments which required the use of visually normal observers. We felt that individuals who habitually wore corrective lenses would probably not meet the stringent visual requirements which we had in mind. Therefore, to save time for both the experimenters and interested participants, we sought college student volunteers by distributing a set of posters which clearly specified: "Subjects with normal vision needed. Must not wear glasses or contact lenses." The individuals who arrived in our laboratory therefore had avowedly "normal" uncorrected visual acuity.

Once in the laboratory, each observer was screened. Standard Snellen acuities were determined for each eye at both the near and the far convergence positions, using a Keystone telebinocular. This procedure produced a surprising finding. We found that 15 of our 111 supposedly visually normal volunteers demonstrated visual acuities below the 20/40 range. One should keep in mind that a Snellen acuity of $20 / 40$ or below is the usually accepted legal definition of impaired vision. Thus, in this sample, $13.5 \%$ of the subject volunteers believed that their vision was normal when it actually fell within the impaired range. The situation is actually worse when one considers the total pattern of test responses. Considering all of the screening tests (right and left eye testing at near and far distances) we found that $42 \%$ of the sample responded with an acuity score between $20 / 30$ and $20 / 40$ on at least one of these four measures.

The poor performance of our subjects on these standard acuity tests caused us to wonder about whether other researchers were pretesting the acuity of their subjects in experiments involving visual processes. To answer this question, we surveyed 264 articles in four major journals, Perception \& Psychophysics, Vision

This research was supported by grants from the Medical Research Council of Canada (MA-5382) and the National Research Council of Canada (A 9783). Reprint requests should be sent to the first author at the Department of Psychology, University of British Columbia, 2075 Wesbrook Place, Vancouver, Canada V6T 1 W5.
Research, The American Journal of Psychology, and The Journal of Experimental Psychology. All of the surveyed articles were recent publications (1972-1974) dealing with various aspects of visual functioning. Areas of investigation included pattern recognitiion, contour detection, and contrast sensitivity, to name a few. Articles dealing with special populations, such as the color blind, were excluded from our sample.

The result of this review produced quite a shock. We found that 189 of these articles (that is, 71.6\%) did not even mention the visual acuity of the subjects involved. No visual pretesting was specified, nor was there any statement which indicated that the vision of the subjects was presumed to be within the normal range. Another 42 articles $(15.9 \%)$ stated blandly that observers had "normal" vision, but there was no mention of any visual screening procedures. The most frequently occurring phrase in this group is that observers had "normal or corrected" vision. This statement leaves a good deal of ambiguity about whether acuity was actually measured. There seems to be the implication that individuals without glasses were taken to be visually normal and those with glasses were assumed to be adequately corrected when their glasses were in place. Six other studies (2.3\%) specified that observers without corrective lenses were recruited. As we have seen, the results of our own recruiting indicate that such a specification is not an adequate guarantee of normal visual acuity. Twenty-four papers $(9.1 \%)$ seem to indicate that some testing procedure was used, although specifics are not given. Here we find that observers are reported as being " $20 / 20$," " $20 / 30$ or better," "emetropic" or "lacking in astigmatism." In three cases, the actual refractive correction used in the subject's eye was specified. In only eight cases (3\%), were the actual acuity tests used specified. These employed the Keystone telebinoculars, the Bausch \& Lomb orthorater, and, in one instance, a Snellen chart.

One might be tempted to feel that we are highlighting an editorial problem rather than an experimental issue. Clearly, all of these authors are experts in vision, hence 
it must be the case that they have prescreened their observers. If this is so, how does one account for the absence of even a cursory note on the visual abilities of their observers in nearly $72 \%$ of the studies which we reviewed? It does not seem reasonable to blame space limitation in journals, since the specification of a subject's visual state and the procedures used to ascertain it would only take a sentence or two. Rather, the impression that one is left with is of a researcher who casually sits his subject down in front of his optical system, projector, or tachistoscope and begins to run his experiment. There is not even a momentary pause to have an observer read a line off of a Snellen chart hung on the wall at some appropriate distance. The researcher is making the presumption that any observer who does not wear glasses is visually normal and any observer who does wear glasses has been corrected to normal when wearing them.

The upshot of all this in terms of the data obtained is obvious. Elevated thresholds for contour and increased reaction times to tachistoscopically presented stimuli can result from the inherent optical degradation in a poorly refracted subject. Such refractive errors may also cause distortions in apparent shape in the direction of increased symmetry or, alternatively, cause an increase in the magnitude of geometric illusions which involve converging lines. At the very least, we must suffer with increased noise and variability in our sample due to acuity deficits in some observers.

The solutions to this situation are simple. If the problem is an editorial one, then authors should be required to specify that their observers were tested and shown to be within the visually normal range. A brief specification of the test used would also be desirable. If observers are not being tested, our own experience indicates that some sort of acuity testing should be instituted prior to the experiment. It need not be elaborate; Snellen acuity, measured at the distance that the stimuli are to be presented, should suffice. Since we continually make extrapolations about the performance of the visually normal eye on the basis of data from our samples, it does not seem to be unrealistic to ask for some guarantee that our observers actually comprised a visually normal group.

(Received for publication February 22, 1975.) 